Computing and Informatics, Vol. 39, 2020, 246 263, doi: 10.31577/cai_2020_1-2 246

\title{
ADAPTIVE FAULT DIAGNOSIS OF MOTORS USING COMPREHENSIVE LEARNING PARTICLE SWARM OPTIMIZER WITH FUZZY PETRI NET
}

\author{
Xuezhen Cheng, Changan Wang, Jiming LI, Xingzhen BaI \\ College of Electrical Engineering and Automation \\ Shandong University of Science and Technology, Qingdao, China \\ e-mail: xzbai@sdust.edu.cn
}

\begin{abstract}
This study proposes and applies a comprehensive learning particle swarm optimization (CLPSO) fuzzy Petri net (FPN) algorithm, which is based on the CLPSO algorithm and FPN, to the fault diagnosis of a complex motor. First, the transition confidence is replaced by a Gaussian function to deal with the uncertainty of fault propagation. Then, according to the Petri net principle, a competition operator is introduced to improve the matrix reasoning. Finally, a CLPSO-FPN model for motor fault diagnosis is established based on the motor failure mechanism and fault characteristics. The CLPSO algorithm is used to generate the system parameters for fault diagnosis and to improve the adaptability and accuracy of fault diagnosis. This study considers the example of a three-phase asynchronous motor. The results show that the proposed algorithm can diagnose faults in this motor with satisfactory adaptability and accuracy compared with the traditional FPN algorithm. By establishing the system model, the fault propagation process of motors can be accurately and intuitively expressed, thus improving the fault treatment and equipment maintenance of motors.
\end{abstract}

Keywords: Fuzzy Petri net, CLPSO, fault diagnosis, motor, adaptive

Mathematics Subject Classification 2010: 93D21

\section{INTRODUCTION}

A motor is a complex mechanical system that usually comprises multiple functional modules. Owing to the complex correlations among these modules, the 
fault characteristics are uncertain and nonlinear 1]. Either quantitative analysis or qualitative analysis is used for fault diagnosis of motors. At present, the data-driven quantitative analysis method is commonly used to process the fault characteristic signals of the rotor and bearing in a motor system for fault classification 2. Deng et al. 3] proposed a motor bearing fault diagnosis method based on the combination of empirical wavelet transform and Hilbert transform; however, this method ignores the noise interference of low-frequency signals and is prone to misjudgment. Deng et al. 44 combined empirical mode decomposition, fuzzy information, and an improved support vector machine method and used the particle swarm optimization (PSO) algorithm to perform parameter optimization, feature extraction, and accurate classification of rotor fault signals. Quantitative analysis can effectively deal with fault signals. However, in complex motor systems, fault signals are easily affected by the environment and motor modules, and most functional modules cannot extract fault signals. Therefore, quantitative analysis cannot easily satisfy the fault diagnosis requirements of motor systems.

Qualitative analysis can be used to establish a system model by using internal knowledge of the system [5]. For example, the Petri net method can be used for graphical and mathematical modeling. In recent years, studies worldwide have used Petri nets to deal with discrete event sequences, concurrency, and conflict relationships [6, 7]. Therefore, Petri nets are increasingly being used for fault diagnosis. Sheng et al. 8] defined the probability transition method of Petri nets for dealing with the uncertainty in the fault propagation process, thereby overcoming the disadvantage that Petri nets only focus on the previous state of places in the fault diagnosis process and that their probability transition mode is not adaptive. Cheng et al. 9] proposed the concept of fuzzy fault Petri net and its modeling method to overcome the difficulty of dealing with the uncertainty of fault information in the fault diagnosis process. Zhang et al. [10] conducted a rigorous mathematical investigation of a fuzzy Petri net (FPN) and proposed a matrix reasoning process, thereby providing a theoretical foundation for applying FPNs. However, the acquisition of its initial weight value still relies on expert experience and has poor adaptability. The neural fuzzy Petri net (NFPN) concept [11] can effectively improve the algorithm's adaptability; however, it cannot satisfy the fault diagnosis requirements of complex motor systems.

To improve the poor accuracy and adaptability of traditional FPN fault diagnosis methods, this study uses the comprehensive learning particle swarm optimization (CLPSO) algorithm to optimize the FPN algorithm and improve the traditional FPN reasoning method. Further, a fault-diagnosis method based on CLPSO-FPN is proposed. The main contributions of this study are as follows:

- A new competition operator is proposed to solve the competition relationship between different modules in complex systems and the matrix reasoning process of the algorithm is optimized. 
- A new representation of transition confidence is proposed, which uses a Gauss function instead of traditional transition confidence and reflects the impact of transition on its output places through a transition influence factor.

- A CLPSO algorithm is used to generate system parameters that reflect the relationship between different modules.

\section{CLPSO-FPN}

\subsection{FPN}

A Petri net can be used to study a network structure based on the known logical relationships between inputs and outputs in a system. In the structure diagram of a Petri net, circles, strips, and directed arcs respectively represent places, transitions, and the relationships between places and transitions. Places represent resources or conditions, and transitions represent events or actions. Figure 1 shows the structure diagram of a Petri net [12].

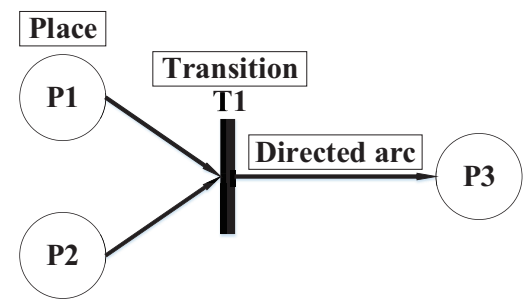

Figure 1. Structure diagram of Petri net

The traditional Petri net with different FPNs uses the place value between $[0,1]$ instead of the token value, and defines the threshold as the condition of transition trigger. The FPN is defined as an 8-tuple, as follows [13].

$$
F P N=(P, T, I, O, M, W, \alpha, \lambda)
$$

where:

- $P=\left\{p_{1}, p_{2}, \ldots, p_{n}\right\}, P$ represents a set of places.

- $T=\left\{t_{1}, t_{2}, \ldots, t_{m}\right\}, T$ represents a set of transitions.

- $I=\left(\delta_{i j}\right)_{(n \times m)}$ is an input matrix describing the mapping of transitions to places. For the input matrix element $\delta_{i j}=\{0,1\}$. If $P_{i}$ is the input of $t_{j}, \delta_{i j}=1$. If $P_{i}$ is not an input of $t_{j}, \delta_{i j}=0 . i=1,2, \ldots, n$ and $j=1,2, \ldots, m$.

- $O=\left(\gamma_{i j}\right)_{(n \times m)}$ is an output matrix describing the mapping of places to transitions. For the output matrix element $\gamma_{i j}=\{0,1\}$. If $t_{j}$ is the input of $P_{i}$, $\gamma_{i j}=1$. If $t_{j}$ is not an input of $P_{i}, \gamma_{i j}=0 . i=1,2, \ldots, n$ and $j=1,2, \ldots, m$. 
- $M=\left(m_{1}, m_{2}, \ldots, m_{n}\right)$ represents a distribution vector of marked places, which is the distribution of tokens in the Petri net.

- $W=\left(\omega_{i j}\right)_{(n \times m)}$ is a weight matrix representing the impact of input places on transitions. $\sum_{i=1}^{n} \omega_{i j}=1$, for $i=1,2, \ldots, n$ and $j=1,2, \ldots, m$.

- $\alpha=\left(\alpha_{1}, \alpha_{2}, \ldots, \alpha_{n}\right)$ is the place value vector, where $\alpha_{i} \in[0,1]$ is the place value.

- $\lambda=\left(\lambda_{1}, \lambda_{2}, \ldots, \lambda_{m}\right)$ is the threshold vector, where $\lambda_{i} \in[0,1]$ is the threshold.

In complex systems, the relationship between different modules is fuzzy and uncertain, therefore, extracted fault information has fuzzy characteristics [14. Studies [15, 16] that combined fuzzy theory and Petri nets to obtain an FPN validated that it could be used for the fault diagnosis of motor systems.

\subsection{CLPSO}

The PSO algorithm can be used to find an optimal solution by simulating cooperation and information transfer among individuals in a group. It mainly includes two elements: speed and position [17, 18. Each particle position represents a possible solution to the equation, and the speed represents the direction and step size of position movement.

When the PSO algorithm is updated, each particle's position and speed are randomly generated. Then, individuals in the group update their individual speeds by judging the group's global and local optimal positions to search for the global optimal position and thereby achieve the purpose of optimization [19].

However, in complex motor systems, the nonlinearity and complexity of the interrelationships between modules lead to large differences between dimensional parameters in particles. If particles are updated uniformly, the difference between dimensional parameters will be lost, resulting in local optimal conditions. The CLPSO algorithm enables independently optimizing different dimensional parameters in particles. It can effectively solve the problem of different dimensional parameters in particles and affords improved optimization ability. The algorithm is comparable to the BP neural network algorithm, which has a strong global optimization ability and prevents the problem of missing faults during fault diagnosis of the motor system. Compared with the PSO algorithm, it has an improved local optimization ability. It can prevent the problems of fault misjudgment during fault diagnosis of each module of the system. Therefore, it is more suitable for complex motors than the traditional optimization algorithm [20].

The CLPSO speed updated formula is as follows:

$$
V_{i}^{d}=\omega * V_{i}^{d}+c * \operatorname{rand}_{i}^{d} *\left(X_{g}^{d}-X_{i}^{d}\right)
$$

The CLPSO position updated formula is as follows:

$$
X_{i}^{d}=X_{i}^{d}+V_{i}^{d}
$$


where $\omega$ is the inertia constant and is a real number in the range of $[0,1], c$ is a learning factor and is a real number in the range of $[0,2], \operatorname{rand}_{i}^{d}$ is a random number in the range of $[0,1], V_{i}^{d}$ and $X_{i}^{d}$ are respectively the speed and position of the $d^{\text {th }}$ dimension of the $i^{\text {th }}$ particle, and $X_{g}^{d}$ is the particle value of the global optimal position. The error formula is as follows:

$$
E=\frac{1}{2} * \sum_{i=1}^{n}\left(\alpha\left(P_{i}\right)-\alpha^{E}\left(P_{i}\right)\right)^{2}
$$

where $\alpha\left(P_{i}\right)$ and $\alpha^{E}\left(P_{i}\right)$ are respectively the $i^{\text {th }}$ place value obtained by reasoning and by Bayesian treatment.

\subsection{CLPSO-FPN}

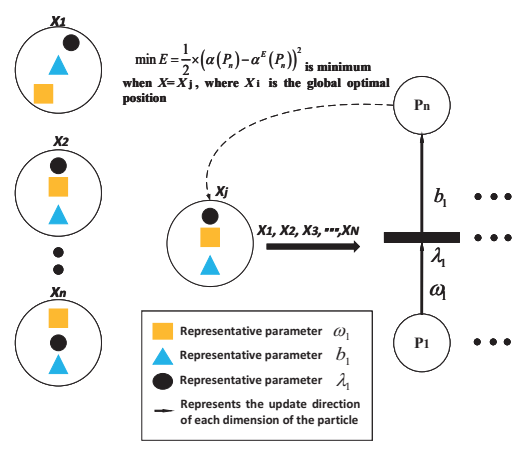

a)

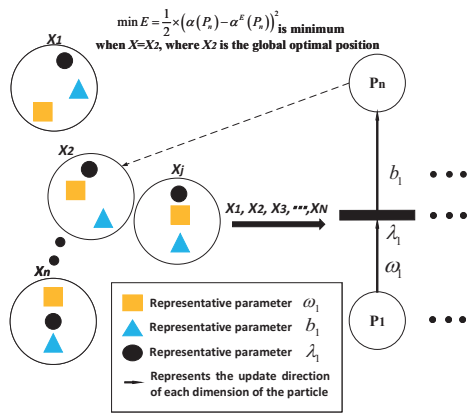

c)

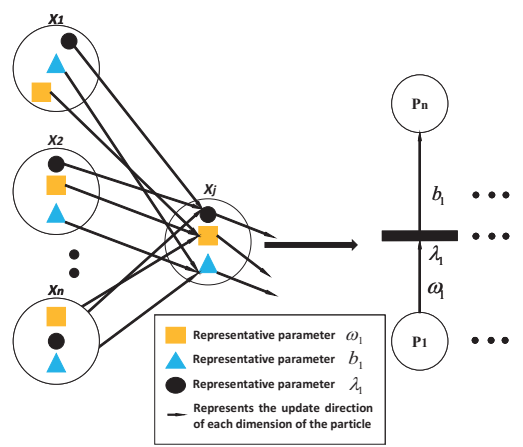

b)

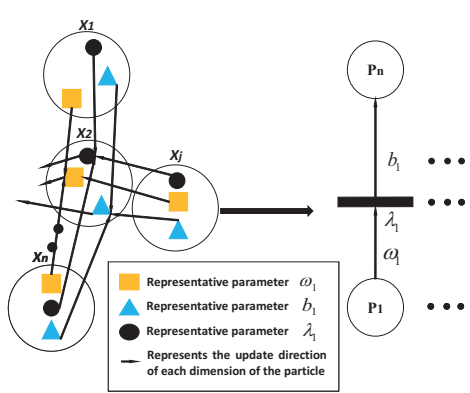

d)

Figure 2. The CLPSO-FPN algorithm parameter generation process 
Figure 2 shows the parameter generation process of the proposed CLPSO-FPN algorithm. The steps in this process are outlined below.

- The CLPSO algorithm randomly generates $n$ particles, each of which includes $\omega_{1}, b_{1}$, and $\lambda_{1}$. The parameters of the $n$ particles are input into the FPN, and $E$ for place $P_{n}$ is obtained by supervised learning. Then, the minimum error minE is obtained, and the corresponding particle $X_{j}$ is the global optimal value in the next iteration process.

- The particles in the global optimal position are determined, and respective dimensional parameters in other particles are updated to the corresponding parameter directions in $X_{j}$. Further, $X_{j}$ randomly updates the positions of the respective dimensional parameters.

- The $n$ updated particles are input into the FPN to obtain particles with minimum error minE.

- The position of $n$ particles is updated until the smallest error found in the end of the iteration is the optimal particle.

The CLPSO-FPN algorithm is mainly used for fault diagnosis of motor systems. First, based on the FPN principle, the system model is established according to the fault operation mechanism and fault characteristics of the system. Second, the CLPSO algorithm is used to randomly generate the weights, threshold, and transition influencing factor. Third, supervised learning is performed according to the actual place value to obtain the system parameter set of the FPN model with the smallest error. Finally, fault diagnosis of the motor system is performed according to the FPN fault diagnosis principle. This method can generate adaptive system parameters for different fault models, and effectively solve the problems of poor accuracy and adaptability of fault diagnosis in the traditional FPN algorithm that are caused by the assignment of system parameters based on experts' experience.

Next, complex motor systems involve multiple mapping relationships between the physical structure of the device and the faults [21, 22]. According to the Petri net principle, this study introduces competition operators, big operators, and a direct multiplication operator. The ability of the CLPSO-FPN algorithm to deal with the competition between different modules in complex motor systems is improved by the operator characteristics, and the matrix reasoning process of the algorithm is optimized.

- $\nabla: C=\nabla A$, where $A$ is an $(m \times n)$-dimensional matrix and $C$ is an $n$-dimensional vector, such that $c_{j}=\max _{1 \leq i \leq m}\left(a_{i j}\right)$.

- $\oplus: C=A \oplus B$, where $A, B$, and $C$ are all $(m \times n)$-dimensional matrices, such that $c_{i j}=\max _{1 \leq i \leq m}\left(a_{i j}, b_{i j}\right)$.

- $\otimes: C=A \otimes b$, where $A$ and $C$ are $(m \times n)$-dimensional matrices and $b$ is an $m$-dimensional vector, such that $c_{i j}=a_{i j} \times b_{i}$. 
Finally, to solve the nonlinear characteristics of the interrelationships among different modules in complex motor systems, a Gaussian function is used to replace the transition confidence, and the transition influencing factor reflects the influence of the transition on the output place. According to the above principle, CLPSO-FPN is defined as a 13-tuple as follows [23:

$$
S_{\text {clpso-fpn }}=(P, T, I, O, M, W, \alpha, \lambda, B, X, N, D, K)
$$

where

- $B=\left(b_{1}, b_{2}, \ldots, b_{m}\right)$ is a transition influence factor vector representing the ability to influence the transition on output place,

- $X=\{W, B, \lambda\}$ is the particle value,

- $N$ is the number of particles,

- $D$ is the number of dimension,

- $K$ is the number of iterations.

\section{CLPSO-FPN FAULT DIAGNOSIS}

According to the CLPSO-FPN principle, the following reasoning calculations are performed for fault diagnosis.

\subsection{Transition Trigger Reasoning}

$H_{k}=\left(h_{1}, h_{2}, \ldots, h_{m}\right)$ is an $m$-dimensional vector that is the sum of the marked place value and the corresponding weight product.

$$
H_{k}=\left(\alpha_{k} * M_{k}\right) \otimes W .
$$

To determine the transition trigger, the Sigmoid function is as follows:

$$
s=1 /(1+\exp (-z(h-\lambda))) .
$$

$S_{k}=\left(s_{1}, s_{2}, \ldots, s_{m}\right)$ is the pre-trigger matrix of the transition, where $z$ is plus infinity and $\lambda$ is the transition threshold. If $h \geq \lambda$, then $s(h)=1$, otherwise $s(h)=0$.

\subsection{Fault Propagation Reasoning}

The NFPN's forward dynamic fault reasoning process reflects the propagation direction of the system fault. Further, tokens reflect the occurrence of system faults. As a transition is triggered, a token is passed from the input place to the output place [23]. The fault propagation reasoning formula is as follows:

$$
M_{k+1}=M_{k} \oplus\left[\frac{1}{1+\exp \left[-z\left(\left(S_{k} \cdot O^{T}\right)-1\right)\right]}\right] .
$$




\subsection{Place Value Reasoning}

To judge the place value in the CLPSO-FPN model, based on the traditional FPN, this study uses the Gaussian function $1 / \exp \left(-10 b \times(x-1)^{2}\right)$ to replace the transition confidence, reflect the influence of the change in output place through the change transition influence factor, and reflect the influence of transition on the output place through the transition influence factor. For dealing with competitive characteristics in the fault diagnosis process of Petri net, the competition operator is introduced, and the matrix reasoning method is optimized. The place value reasoning is as follows:

$$
\alpha_{k+1}=\alpha_{k} \oplus\left[\nabla\left(\left(\frac{X_{k}}{e^{10 B_{k} *\left(X_{k}-1\right)^{2}}}\right)^{T} \otimes O^{T}\right)\right],
$$

according to the requirements of the NFPN algorithm for fault diagnosis, when the reasoning is complete.

\subsection{CLPSO-FPN Fault Diagnosis Progress}

In the traditional FPN-based fault diagnosis method, system parameters are usually assigned based on experts' experience, and therefore, the accuracy and adaptability of fault diagnosis are poor. To solve these problems, this study uses the CLPSO algorithm to randomly generate the weights, threshold, and transition influencing factor as dimensional parameters in particles. The update direction of each dimensional parameter of other particles is determined by finding the global optimal particle. Then, according to the comprehensive learning strategy, each dimensional parameter is differentiated, and its speed value and parameter value in the particle are updated. This method can perform differential training according to the characteristics of different parameters and can find the system parameter set under the optimal conditions. Finally, using the parameter set in the particle for fault diagnosis reasoning, the probability of each module failure occurs. The CLPSO-FPN fault diagnosis process is in Figure 3.

\section{DATA PROCESSING AND MODEL CONSTRUCTION}

In this study, a three-phase asynchronous motor is taken as an example to conduct model construction and data simulation. According to the data in [9], failure mode analysis and FPN are combined to analyze fault data and to establish a fault relation table that conforms to the complex motor system [24]. Table 1] shows the training sample set for the complex motor system. Further, Figure 4 shows the CLPSO model established for this motor based on its structure and fault analysis as well as the data in Table 1 .

For this CLPSO-FPN model, this study uses a Bayesian method to process the fault data and combines the fault propagation mode of the motor system to determine the actual place value [25]. This method could effectively and accurately 


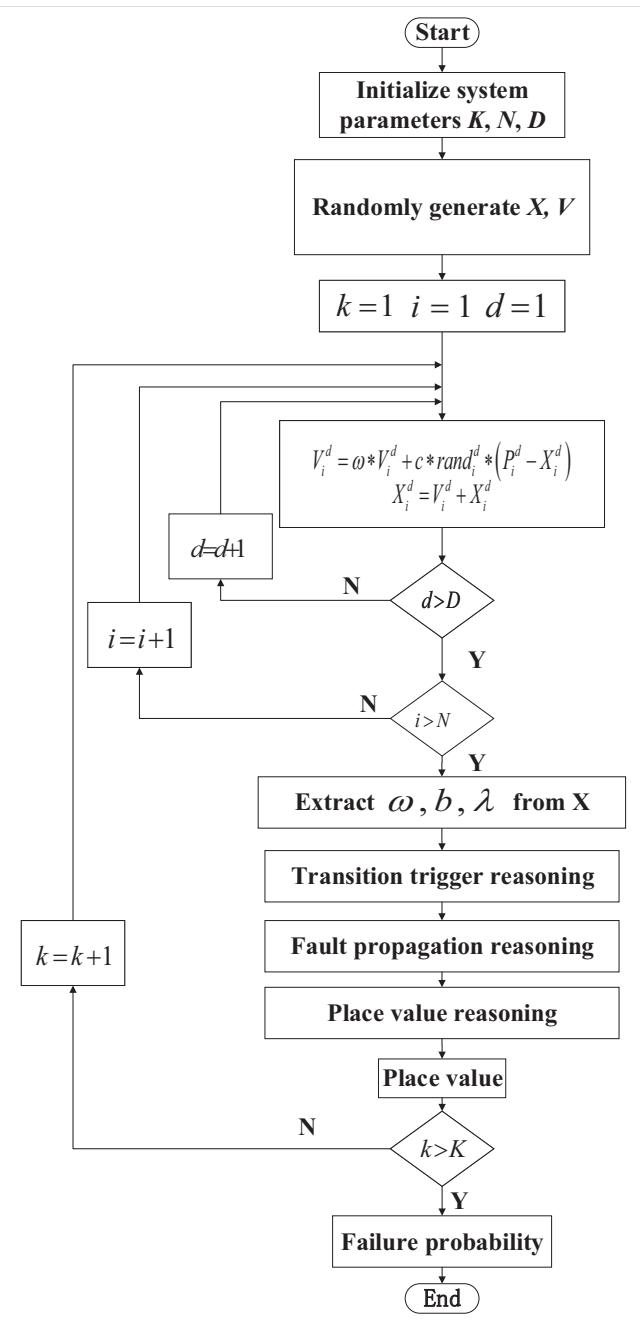

Figure 3. The CLPSO-FPN fault diagnosis process

convert knowledge and experience to rules, this is helpful in solving the problem of empirical assignment of parameters and in achieving accurate fault diagnosis of the motor. 


\section{METHOD IMPLEMENTATION AND VERIFICATION}

\subsection{Initial Value Determination}

In this study, the "motor winding insulation burned" is taken as an example. According to the place value of the actual place, the CLPSO-FPN algorithm is used for supervised learning. The actual place values are shown in Table 2. When $K=1000$ and $N=40$, the error curve of the CLPSO algorithm is obtained under different numbers of iterations, as shown in Figure 5. The system parameters set is shown in Table 3 .

\subsection{Verification of the Method}

To verify the optimization performance of the CLPSO algorithm for FPN fault diagnosis, the place values and accuracies obtained using the CLPSO, PSO, and back-propagation (BP) algorithms are compared in Figure 6 and Figure 7. These figures show that the accuracy of the place value obtained using the BP algorithm is poor, whereas that obtained using the PSO algorithm is satisfactory; however, the local optimization ability is poor. By contrast, the accuracy of the place value obtained using the CLPSO algorithm is high, and the overall optimization ability is satisfactory. The result proves that the algorithm is more suitable for fault diagnosis of complex motor systems than PSO and BP algorithms.

The effectiveness and accuracy of the CLPSO-FPN algorithm for the fault diagnosis of three-phase asynchronous motors is verified for three different fault conditions through comparisons with two previous algorithms [9, 12, the results are shown in Table 4.

These results indicate that the FFPN and NFPN algorithms show misjudgments in the motor fault diagnosis process. By contrast, the proposed CLPSO-FPN fault diagnosis method can accurately determine the "motor winding insulation burned" problem and can effectively solve the problems of fault diagnosis and misjudgment. Further, it shows better accuracy than the FFPN and NFPN algorithms. Therefore, it can satisfy the fault diagnosis requirements of complex motor systems and shows higher accuracy and adaptability than the traditional FPN fault diagnosis method.

\section{CONCLUSIONS}

With the motor developing toward large-scale, complication and integrated direction, which leads to the traditional fault diagnosis method, it is difficult to meet the fault diagnosis requirements of the motor system. To solve these problems, this study proposes and applies a CLPSO-FPN algorithm to the fault diagnosis of a complex motor. In the proposed fault diagnosis method the CLPSO-FPN model of motor, using a reasoning process to diagnose fault, is established. Then a Gaussian function is used to replace the traditional transition confidence in the CLPSO-FPN 


\begin{tabular}{|c|c|c|c|}
\hline Code & Meaning & Code & Meaning \\
\hline$P_{1}$ & $\begin{array}{l}\text { Phase winding resistance } \\
\text { becomes smaller }\end{array}$ & $P_{23}$ & $\begin{array}{l}\text { Motor overload or } \\
\text { irregular impact load }\end{array}$ \\
\hline$P_{2}$ & $\begin{array}{l}\text { Rotor winding short } \\
\text { circuit }\end{array}$ & $P_{24}$ & Excessive bearing wear \\
\hline$P_{3}$ & Overload of motor & $P_{25}$ & Motor holding shaft \\
\hline$P_{4}$ & Fuse melt failure & $P_{26}$ & $\begin{array}{l}\text { Bearing locking device } \\
\text { failure }\end{array}$ \\
\hline$P_{5}$ & $\begin{array}{l}\text { Shaft seal ring } \\
\text { structure damage }\end{array}$ & $P_{27}$ & Rotor core deformation \\
\hline$P_{6}$ & $\begin{array}{l}\text { Oil seal material } \\
\text { overheated }\end{array}$ & $P_{28}$ & $\begin{array}{l}\text { Magnetic slot wedge } \\
\text { fracture or detachment }\end{array}$ \\
\hline$P_{7}$ & $\begin{array}{l}\text { Seal surface axis } \\
\text { roughness value is too large }\end{array}$ & $P_{29}$ & $\begin{array}{l}\text { Rotor winding open } \\
\text { circuit }\end{array}$ \\
\hline$P_{8}$ & $\begin{array}{l}\text { Temperature is too } \\
\text { high }\end{array}$ & $P_{30}$ & $\begin{array}{l}\text { Junction box joint } \\
\text { loosening }\end{array}$ \\
\hline$P_{9}$ & $\begin{array}{l}\text { Exciting current is } \\
\text { too large }\end{array}$ & $P_{31}$ & $\begin{array}{l}\text { Poor contact of the } \\
\text { power control loop switch }\end{array}$ \\
\hline$P_{10}$ & $\begin{array}{l}\text { A phase current } \\
\text { is too large }\end{array}$ & $P_{32}$ & $\begin{array}{l}\text { Rotor winding mechanical } \\
\text { failure }\end{array}$ \\
\hline$P_{11}$ & Rotational speed abnormality & $P_{33}$ & $\begin{array}{l}\text { The central line of motor } \\
\text { is not consistent with the } \\
\text { center line of shearer }\end{array}$ \\
\hline$P_{12}$ & $\begin{array}{l}\text { Loss of phase } \\
\text { voltage }\end{array}$ & $P_{34}$ & $\begin{array}{l}\text { Axial movement of } \\
\text { rotor }\end{array}$ \\
\hline$P_{13}$ & $\begin{array}{l}\text { Bearing is thermally } \\
\text { expanded }\end{array}$ & $P_{35}$ & $\begin{array}{l}\text { Spring attachment device } \\
\text { failure }\end{array}$ \\
\hline$P_{14}$ & $\begin{array}{l}\text { Oil entering the } \\
\text { motor }\end{array}$ & $P_{36}$ & Scratching of motor \\
\hline$P_{15}$ & $\begin{array}{l}\text { Bearing is thermally } \\
\text { expanded }\end{array}$ & $P_{37}$ & Stator current increase \\
\hline$P_{16}$ & Motor overheating & $P_{38}$ & Excessive pressure drop \\
\hline$P_{17}$ & $\begin{array}{l}\text { Motor in Open-phase } \\
\text { State }\end{array}$ & $P_{39}$ & $\begin{array}{l}\text { Excessive operational shock } \\
\text { of motor }\end{array}$ \\
\hline$P_{18}$ & $\begin{array}{l}\text { Motor rotation is } \\
\text { abnormal or card machine }\end{array}$ & $P_{40}$ & $\begin{array}{l}\text { Excessive noise of } \\
\text { bearing }\end{array}$ \\
\hline$P_{19}$ & Motor insulation aging & $P_{41}$ & $\begin{array}{l}\text { The motor turns weak or } \\
\text { does not rotate and buzz }\end{array}$ \\
\hline$P_{20}$ & $\begin{array}{l}\text { Reduction of lubricating } \\
\text { oil content }\end{array}$ & $P_{42}$ & $\begin{array}{l}\text { Motor running abnormal } \\
\text { sound }\end{array}$ \\
\hline$P_{21}$ & $\begin{array}{l}\text { Curved ring and axis } \\
\text { hole produce friction }\end{array}$ & $P_{43}$ & Motor failure \\
\hline$P_{22}$ & $\begin{array}{l}\text { Motor winding insulation } \\
\text { burned }\end{array}$ & & \\
\hline
\end{tabular}

Table 1. Event table of places 


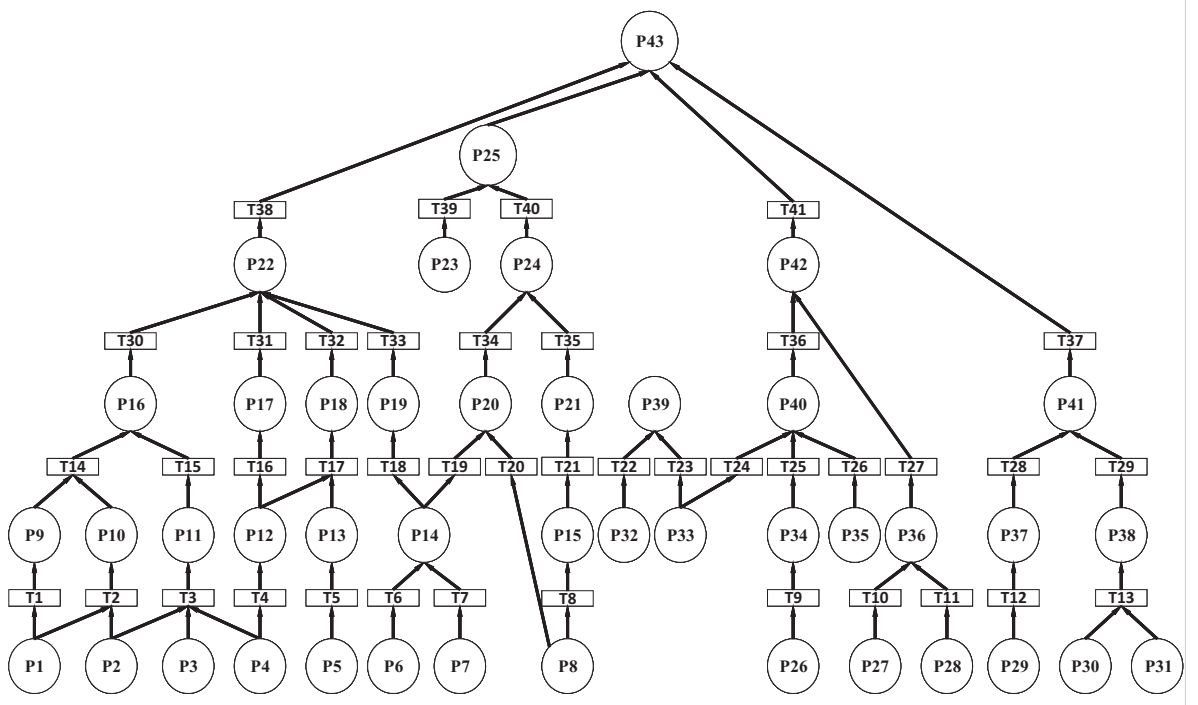

Figure 4. CLPSO-FPN model of three phase asynchronous motor

algorithm to reflect the influence of transition on the output place and a competition operator combined with a sigmoid function is proposed to determine the transition trigger reasoning. This method optimizes the matrix reasoning process compared with the traditional FPN algorithm. Finally, using the CLPSO algorithm to generate system parameters can effectively solve the problem of human's subjective

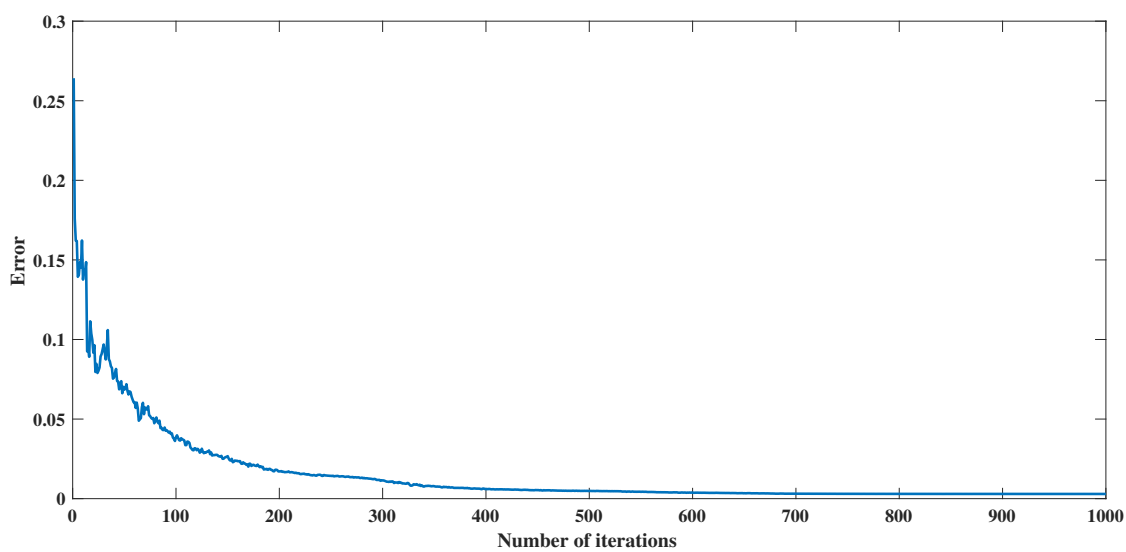

Figure 5. CLPSO iteration number error curve 


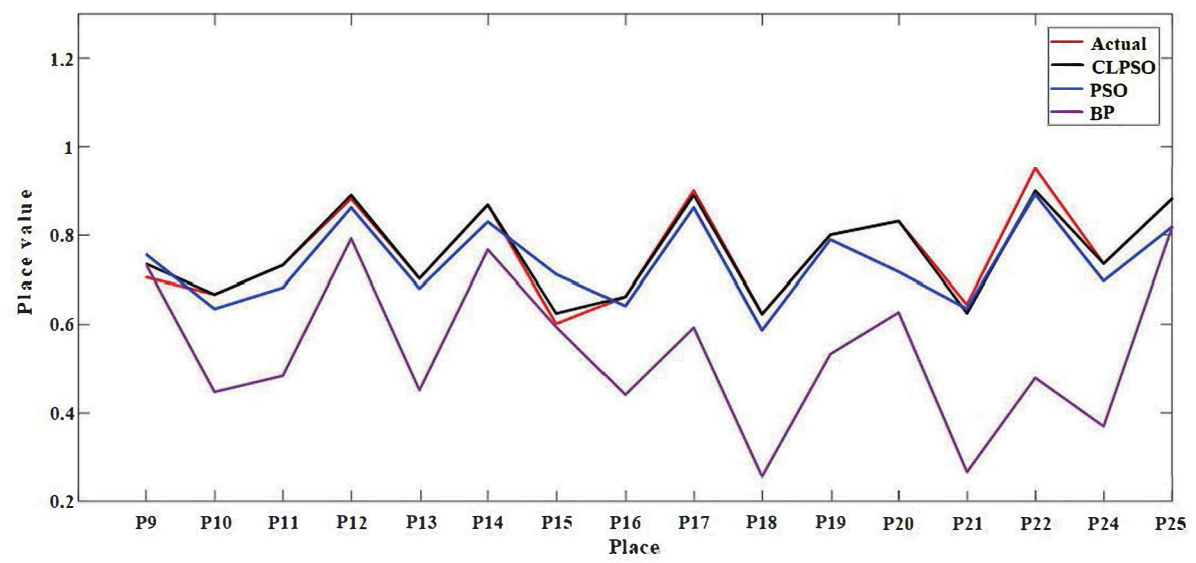

Figure 6. Place value curve

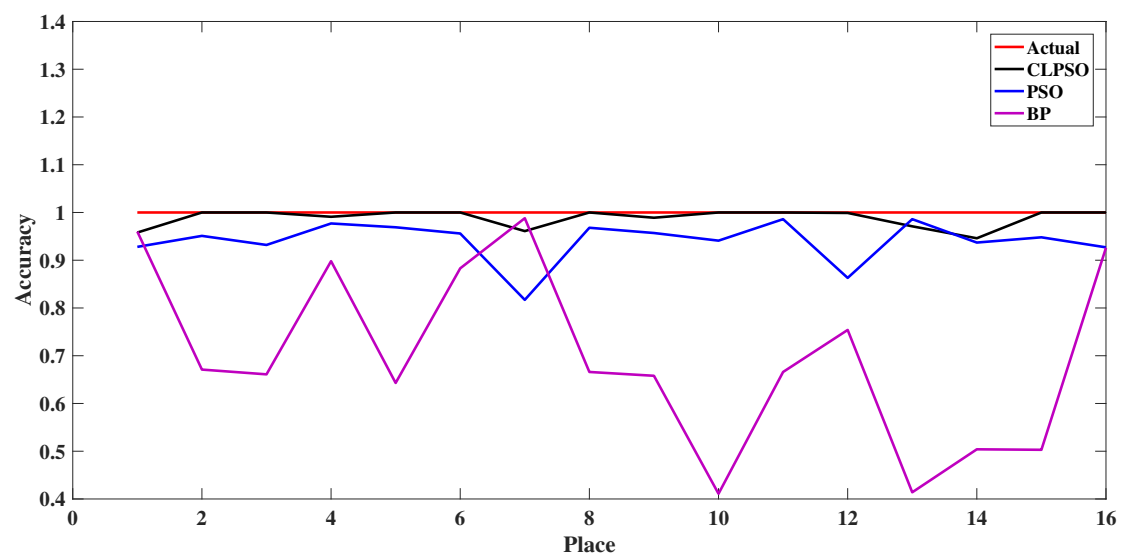

Figure 7. Accuracy curve

\begin{tabular}{lrlr}
\hline Code & Actual Place Value & Code & Actual Place Value \\
\hline$P_{9}$ & 0.7051 & $P_{17}$ & 0.9001 \\
$P_{10}$ & 0.6670 & $P_{18}$ & 0.6230 \\
$P_{11}$ & 0.7320 & $P_{19}$ & 0.8002 \\
$P_{12}$ & 0.8821 & $P_{20}$ & 0.8311 \\
$P_{13}$ & 0.7020 & $P_{21}$ & 0.6438 \\
$P_{14}$ & 0.8682 & $P_{22}$ & 0.9510 \\
$P_{15}$ & 0.7320 & $P_{24}$ & 0.7351 \\
$P_{16}$ & 0.6620 & $P_{25}$ & 0.8812 \\
\hline
\end{tabular}

Table 2. Actual place values 


\begin{tabular}{lccrcc}
\hline \multicolumn{2}{c}{ Weights } & \multicolumn{2}{c}{ Transition } & \multicolumn{2}{c}{ Threshold } \\
\hline$\omega_{1,1}$ & 1 & $b_{1}$ & 0.635 & $\lambda_{1}$ & 0.488 \\
$\omega_{1,2}$ & 0.519 & $b_{2}$ & 0.557 & $\lambda_{2}$ & 0.526 \\
$\omega_{2,2}$ & 0.481 & $b_{3}$ & 0.391 & $\lambda_{3}$ & 0.498 \\
$\omega_{2,3}$ & 0.370 & $b_{4}$ & 0.517 & $\lambda_{4}$ & 0.483 \\
$\omega_{3,3}$ & 0.300 & $b_{5}$ & 0.479 & $\lambda_{5}$ & 0.484 \\
$\omega_{4,3}$ & 0.330 & $b_{6}$ & 0.334 & $\lambda_{6}$ & 0.517 \\
$\omega_{4,4}$ & 1 & $b_{7}$ & 0.347 & $\lambda_{7}$ & 0.524 \\
$\omega_{5,5}$ & 1 & $b_{14}$ & 0.485 & $\lambda_{14}$ & 0.464 \\
$\omega_{6,6}$ & 1 & $b_{15}$ & 0.592 & $\lambda_{15}$ & 0.510 \\
$\omega_{7,7}$ & 1 & $b_{16}$ & 0.463 & $\lambda_{16}$ & 0.447 \\
$\omega_{9,14}$ & 0.536 & $b_{17}$ & 0.437 & $\lambda_{17}$ & 0.495 \\
$\omega_{10,14}$ & 0.464 & $b_{18}$ & 0.298 & $\lambda_{18}$ & 0.543 \\
$\omega_{11,15}$ & 1 & $b_{30}$ & 0.526 & $\lambda_{30}$ & 0.455 \\
$\omega_{12,16}$ & 1 & $b_{31}$ & 0.541 & $\lambda_{31}$ & 0.465 \\
$\omega_{12,17}$ & 0.392 & $b_{32}$ & 0.480 & $\lambda_{32}$ & 0.486 \\
$\omega_{13,17}$ & 0.608 & $b_{33}$ & 0.404 & $\lambda_{33}$ & 0.456 \\
$\omega_{14,18}$ & 1 & & & & \\
$\omega_{16,30}$ & 1 & & & & \\
$\omega_{17,31}$ & 1 & & & & \\
$\omega_{18,32}$ & 1 & & & & \\
$\omega_{19,33}$ & 1 & & & & \\
\hline
\end{tabular}

Table 3. Set of system parameters

factor effectively due to the assignment of parameters based on experts' experience. Therefore, the results indicate that the method can effectively improve the accuracy and adaptability of fault diagnosis, which can improve the fault treatment and equipment maintenance of motors.

Several interesting research topics are to be considered in the future, for example,

1. the online fault diagnosis problem for motor and wireless localization systems [26],

2. the online fault prediction problem for wireless sensor networks [27].

\section{REFERENCES}

[1] Chen, X.-Bai, X.-Zhang, Q.: Micro Grid Fault Diagnosis Based on Redundant Embedding Petri Net. Systems Science and Control Engineering, Vol. 6, 2018, No. 3, pp. 289-296, doi: $10.1080 / 21642583.2018 .1554801$.

[2] Bai, X.-WAng, Z.-Zou, L.-AlsaAdi, E. F.: Collaborative Fusion Estimation over Wireless Sensor Networks for Monitoring CO2 Concentration in a Greenhouse. Information Fusion, Vol. 42, 2018, pp. 119-126, doi: 10.1016/j.inffus.2017.11.001. 


\begin{tabular}{|c|c|c|c|c|c|c|}
\hline Method & $\begin{array}{l}\text { Cause } \\
\text { of Fault }\end{array}$ & $\begin{array}{l}\text { Diagnosed } \\
\text { Fault }\end{array}$ & Fault & $\begin{array}{c}\text { Actual } \\
\text { Failure } \\
\text { Probability }\end{array}$ & $\begin{array}{l}\text { Diagnostic } \\
\text { Failure } \\
\text { Probability }\end{array}$ & Accuracy \\
\hline \multirow{3}{*}{ FFPN } & $P_{4}$ & $P_{12}, P_{17}, P_{22}$ & $P_{22}$ & 0.8910 & 0.4270 & $47.9 \%$ \\
\hline & $P_{1}, P_{3}$ & $P_{9}$ & $P_{22}$ & 0.4121 & 0 & 0 \\
\hline & $P_{1}, P_{2}, P_{5}$ & $\begin{array}{c}P_{9}, P_{10}, P_{13} \\
P_{16}\end{array}$ & $P_{22}$ & 0.5120 & 0.6020 & $82.4 \%$ \\
\hline \multirow{3}{*}{ NFPN } & $P_{4}$ & $P_{12}, P_{17}, P_{22}$ & $P_{22}$ & 0.8910 & 0.4794 & $53.8 \%$ \\
\hline & $P_{1}, P_{3}$ & $P_{9}, P_{16}$ & $P_{22}$ & 0.4121 & 0 & 0 \\
\hline & $P_{1}, P_{2}, P_{5}$ & $\begin{array}{c}P_{9}, P_{10}, P_{13} \\
P_{16}\end{array}$ & $P_{22}$ & 0.5120 & 0 & 0 \\
\hline \multirow{3}{*}{$\begin{array}{c}\text { CLPSO } \\
\text { FPN }\end{array}$} & $P_{4}$ & $\begin{array}{c}P_{11}, P_{12}, P_{16}, \\
P_{17}, P_{18}, P_{22}\end{array}$ & $P_{22}$ & 0.8910 & 0.8950 & $99.6 \%$ \\
\hline & $P_{1}, P_{3}$ & $\begin{array}{c}P_{9}, P_{10}, P_{16} \\
P_{22}\end{array}$ & $P_{22}$ & 0.4121 & 0.3665 & $88.9 \%$ \\
\hline & $P_{1}, P_{2}, P_{5}$ & $\begin{array}{l}P_{9}, P_{10}, P_{13} \\
P_{16}, P_{18}, P_{22}\end{array}$ & $P_{22}$ & 0.5120 & 0.3666 & $71.6 \%$ \\
\hline
\end{tabular}

Table 4. Fault diagnosis table

[3] Deng, W.-Zhang, S.-Zhao, H.-Yang, X.: A Novel Fault Diagnosis Method Based on Integrating Empirical Wavelet Transform and Fuzzy Entropy for Motor Bearing. IEEE Access, Vol. 6, 2018, pp. 35042-35056, doi: 10.1109/ACCESS.2018.2834540.

[4] Deng, W.-Yao, R.-Zhao, H.-Yang, X.-Li, G.: A Novel Intelligent Diagnosis Method Using Optimal LS-SVM with Improved PSO Algorithm. Soft Computing, Vol. 23, 2019, No. 7, pp. 2445-2462, doi: 10.1007/s00500-017-2940-9.

[5] Lu, Y.-WAng, F.-Jia, M.: Qualitative Simulation and Fuzzy Knowledge Based Fault Diagnosis of Centrifugal Compressor Insufficient Discharge. Acta Automatica Sinica, Vol. 41, 2015, No. 11, pp. 1867-1876, doi: 10.16383/j.aas.2015.c150117 (in Chinese).

[6] Kabir, S.-Papadopoulos, Y.: Applications of Bayesian Networks and Petri Nets in Safety, Reliability, and Risk Assessments: A Review. Safety Science, Vol. 115, 2019, pp. 154-175, doi: 10.1016/j.ssci.2019.02.009.

[7] Bian, L.-Bian, C.: Review on Intelligence Fault Diagnosis in Power Networks. Power System Protection and Control, Vol. 42, 2014, No. 3, pp. 146-153 (in Chinese).

[8] Sheng, S.-Xiao, M.-Zhao, L.-Wen, Y.-Hu, B.: Research on Probability Transition Method for Fault Petri Net. Chinese Journal of Scientific Instrument, Vol. 35, 2014, No. 3, pp. 714-720, doi: 10.19650/j.cnki.cjsi.2014.03.033 (in Chinese).

[9] Cheng, X.-Wang, C.-Yu, Y.-Yi, L.-Chen, Q.: An Approach for ThreePhase Asynchronous Motor Failure Analysis Based on Fuzzy Fault Petri Net. Transactions of China Electrotechnical Society, Vol. 30, 2015, No. 17, pp. 132-139, doi: 10.19595/j.cnki.1000-6753.tces.2015.17.015 (in Chinese).

[10] Zhang, Y.-Zhang, Y.-Wen, F.-Chun, C. Y.-Tseng, C.-Zhang, X.Zeng, F.-Yuan, Y.: A Fuzzy Petri Net Based Approach for Fault Diag- 
nosis in Power Systems Considering Temporal Constraints. International Journal of Electrical Power and Energy Systems, Vol. 78, 2016, pp. 215-224, doi: 10.1016/j.ijepes.2015.11.095.

[11] Cheng, X.-Zhu, X.-Du, Y.-Wang, C.-CaO, M.: High Voltage Circuit Breaker Fault Diagnosis Based on Neural Fuzzy Petri Nets. Transactions of China Electrotechnical Society, Vol. 33, 2018, No. 11, pp. 2535-2544, doi: 10.19595/j.cnki.1000-6753.tces.170533 (in Chinese).

[12] Latsou, C.-Dunnett, S. J.-Jackson, L. M.: A New Methodology for Automated Petri Net Generation: Method Application. Reliability Engineering and System Safety, Vol. 185, 2019, pp. 113-123, doi: 10.1016/j.ress.2018.12.017.

[13] Wang, H.-Jiang, C.-LiaO, S.: Concurrent Reasoning of Fuzzy Logical Petri Nets Based on Multi-Task Schedule. IEEE Transactions Fuzzy Systems, Vol. 9, 2001, No. 3, pp. 444-449, doi: $10.1109 / 91.928740$

[14] DÂmaso, A.-Rosa, N.-Maciel, P.: Using Coloured Petri Nets for Evaluating the Power Consumption of Wireless Sensor Networks. International Journal of Distributed Sensor Networks, Vol. 10, 2014, No. 6, Art. No. 423537, 13 pp., doi: $10.1155 / 2014 / 423537$

[15] Gong, M.-Song, H.-Tan, J.-XIE, Y.-Song, J.: Fault Diagnosis of Motor Based on Mutative Scale Back Propagation Net Evolving Fuzzy Petri Nets. 2017 Chinese Automation Congress (CAC), Jinan, China, 2017, pp. 3826-3829, doi: 10.1109/CAC.2017.8243447.

[16] Kong, D.-Li, H.: Fuzzy Petri Nets and Its Application in Fault Diagnosis of Compressor. Computer Engineering and Design, Vol. 39, 2018, No. 1, pp. 271-275, doi: $10.16208 / \mathrm{j}$.issn1000-7024.2018.01.047 (in Chinese).

[17] Lynn, N.-Suganthan, P. N.: Heterogeneous Comprehensive Learning Particle Swarm Optimization with Enhanced Exploration and Exploitation. Swarm and Evolutionary Computation, Vol. 24, 2015, pp. 11-24, doi: 10.1016/j.swevo.2015.05.002.

[18] Masdari, M.-Salehi, F.-Jalali, M.-Bidaki, M.: A Survey of PSO-Based Scheduling Algorithms in Cloud Computing. Journal of Network and Systems Management, Vol. 25, 2017, No. 1, pp. 122-158, doi: 10.1007/s10922-016-9385-9

[19] Xu, J.-Yan, F.-Yun, K.-Ronald, S.-Li, F.—Guan, J.: Dynamically Dimensioned Search Embedded with Piecewise Opposition-Based Learning for Global Optimization. Scientific Programming, Vol. 2019, 2019, No. 1, Art. No. 2401818, 20 pp., doi: $10.1155 / 2019 / 2401818$.

[20] Wang, J.-GaO, Y.-Liu, W.-Sangaiah, A.K.-Kim, H.: An Improved Routing Schema with Special Clustering Using PSO Algorithm for Heterogeneous Wireless Sensor Network. Sensors, Vol. 19, 2019, No. 3, Art. No. 671, 17 pp., doi: 10.3390/s19030671.

[21] Cheng, Y.-Wang, Z.-Zhang, W.-Huang, G.: Particle Swarm Optimization Algorithm to Solve the Deconvolution Problem for Rolling Element Bearing Fault Diagnosis. ISA Transactions, Vol. 90, 2019, pp. 244-267, doi: 10.1016/j.isatra.2019.01.012.

[22] Bai, X.-WAng, Z.-Sheng, L.-WAng, Z.: Reliable Data Fusion of Hierarchical Wireless Sensor Networks with Asynchronous Measurement for Greenhouse Moni- 
toring. IEEE Transactions on Control Systems Technology, Vol. 27, 2019, No. 3, pp. 1036-1046, doi: 10.1109/TCST.2018.2797920.

[23] Li, J.-Zhu, X.-Cheng, X.: Sensor Fault Diagnosis Based on Fuzzy Neural Petri Net. Complexity, Vol. 2018, 2018, Art. No. 8261549, 11 pp., doi: $10.1155 / 2018 / 8261549$.

[24] LiU, H.-LiU, L.-Lin, Q.-Liu, N.: Knowledge Acquisition and Representation Using Fuzzy Evidential Reasoning and Dynamic Adaptive Fuzzy Petri Nets. IEEE Transactions on Cybernetics, Vol. 43, 2013, No. 3, pp. 1059-1072, doi: 10.1109/TSMCB.2012.2223671.

[25] Tolosana-Calasanz, R.-Bañares, J. Á.-Colom, J.-M.: Model-Driven Development of Data Intensive Applications over Cloud Resources. Future Generation Computer Systems, Vol. 87, 2018, pp. 888-909, doi: 10.1016/j.future.2017.12.046.

[26] Bai, X.-Wang, Z.-Zou, L.-Cheng, C.: Target Tracking for Wireless Localization Systems with Degraded Measurements and Quantization Effects. IEEE Transactions on Industrial Electronics, Vol. 65, 2018, No. 12, pp. 9687-9697, doi: 10.1109/TIE.2018.2813982.

[27] Bai, X.-Liu, L.-CaO, M.-Panneerselvam, J.-Sun, Q.-Wang, H.: Collaborative Actuation of Wireless Sensor and Actuator Networks for the Agriculture Industry. IEEE Access, Vol. 5, 2017, pp. 13286-13296, doi: 10.1109/ACCESS.2017.2725342. 


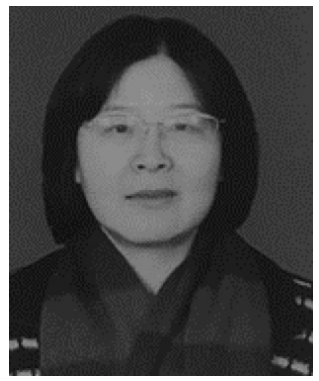

Xuezhen Cheng received her Ph.D. degree in control theory and engineering in 2011 from the Shandong University of Science and Technology, China. She is currently serving as Professor in the School of College of Electrical Engineering and Automation, Shandong University of Science and Technology, China. Her research interests include detection technology and power system automation.

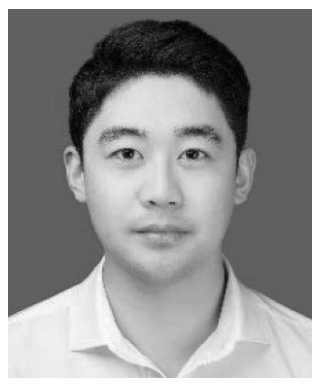

Changan WANG has his M.Sc. degree in electric power system and automation from the Shandong University of Science and Technology. His main research field is fault diagnosis.

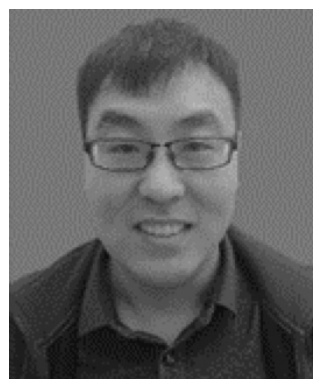

Jiming LI is currently Ph.D. student at the Department of Electrical Engineering and Automation, Shandong University of Science and Technology. His research interests are signal processing, inspection technology and system integration.

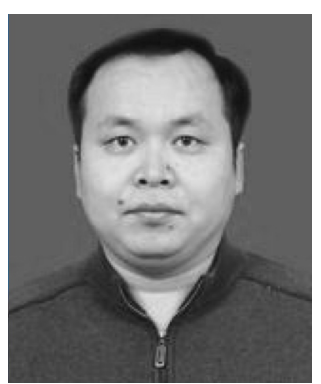

Xingzhen BAI received his Ph.D. degree in computer software and theory from the Tongji University, Shanghai, China, in 2010. He is currently serving as Associate Professor with the College of Electrical Engineering and Automation, Shandong University of Science and Technology. His current research interests include distributed estimation, fault diagnosis, wireless sensor network, and smart grid. 